\title{
Numerical Simulation Study on Influence of Multi-seam Propagation of Hydraulic Fracture in Coal Seam
}

\author{
Zhang Weiqiang, Pu Chunsheng* \\ School of Petroleum Engineering, China University of Petroleum, Qingdao, China \\ Email address: \\ 1223407977@qq.com (Zhang Weiqiang),puchshcup@126.com (Pu Chunsheng) \\ ${ }^{*}$ Corresponding author
}

\section{To cite this article:}

Zhang Weiqiang, Pu Chunsheng. Numerical Simulation Study on Influence of Multi-seam Propagation of Hydraulic Fracture in Coal Seam. Science Discovery. Vol. 8, No. 5, 2020, pp. 107-112. doi: 10.11648/j.sd.20200805.16

Received: September 12, 2020; Accepted: October 9, 2020; Published: October 17, 2020

\begin{abstract}
Multi-stage fracturing technology for horizontal Wells is one of the effective methods of coal seam volume reconstruction at present. This technology can effectively form a volumetric seam network, increase the volume of coal seam segment reconstruction, increase the area of gas seepage channel, and reduce the generation of coal powder. In order to study the mutual interference of multi-fracture extension in hydraulic fracturing of coal seam and to solve the problem of unclear effect of fracturing construction parameters in coal seam at present, this paper, based on the extended finite element method (XFEM), conducts fluid-solid coupling simulation using pore pressure element (C3D8P), and establishes a calculation model of multi-fracture extension in coal seam. This model is used to study the influence of perforating cluster number, cluster spacing and non-uniformity on hydraulic fracture propagation. The calculated results indicate that, with the increase of the number of perforating clusters, the surface stress field is disturbed more seriously by fractures, and the volume of formation reconstruction also increases correspondingly. After comprehensive comparison, it is believed that the optimal fracturing effect can be achieved when two clusters of perforating clusters are used. The larger the cluster spacing, the smaller the inter-fracture interference, and the higher the formation pore pressure during fracturing. The non-uniformity between clusters has a great influence on fracture propagation. When the displacement of the middle cluster is $1 / 4$, the inhibition effect is severe and it is difficult to form effective fracturing fracture. Finally, the model is used to optimize the multi-stage fracturing construction parameters of horizontal well in coal seam, which provides reference and guidance for the design of hydraulic fracturing scheme.
\end{abstract}

Keywords: Horizontal Well, Coal Seam, Hydrofracture, XFEM

\section{煤层水力裂缝多缝扩展影响数值模拟研究}

张伟强, 蒲春生 ${ }^{*}$

中国石油大学 (华东) 石油工程学院, 青岛, 中国

\section{邮箱}

1223407977@qq.com（张伟强）, puchshcup@126.com（蒲春生）

摘要：水平井多段压裂技术是目前煤层体积改造的有效手段之一, 该技术可有效地形成体积缝网, 增加煤层段改造体 积, 增大气体渗流通道面积, 减少煤粉的生成。为研究煤层水力压裂多裂缝扩展相互干扰情况, 针对目前现场煤层压 裂施工参数效果不明确的问题, 本文基于扩展有限元法(XFEM), 运用ABAQUS孔压单元 (C3D8P)进行流固耦合模拟, 建立了煤层多裂缝扩展计算模型。利用该模型研究了射孔簇数、簇间距以及簇间不均匀性对水力裂缝扩展的影响。计 算结果表明：随着射孔簇数的增加, 裂缝对地应力场的干扰现象越严重, 同时地层改造体积也相应增加, 综合对比后 认为两簇射孔时可达到最优的压裂改造效果; 簇间距越大, 裂缝间干扰越小, 压裂时地层孔隙压力越高; 族间不均匀 
性对裂缝扩展影响较大, 当中间簇位于 $1 / 4$ 位置时受到的抑制效果严重, 难以形成有效压裂改造裂缝。最终利用该模型 优化了煤层水平井多段压裂施工参数, 为水力压裂方案的设计提供了参考和指导。

关键词: 水平井, 煤层, 多簇压裂, XFEM

\section{1. 引言}

煤层气是一种非常规性天然气资源, 其作为一种重要 的清洁能源在我国储量巨大 $[1]$ 。但煤层气储层普遍具有低 孔低渗的特点, 所以常规煤层只有经过压裂改造才可形成 连通性较好的有效裂缝系统, 从而实现煤层气的规模生产, 时期具有经济效益。

水平井多段压裂技术是煤层储层物性改造, 形成有效 体积裂缝网络的高效技术之一。针对该技术中裂缝相互作 用机理等问题, 已有多位学者做了相应的研究, Roussel[2] 等建立了多缝压裂的数值模型, 研究了缝间应力变化对裂 缝扩展的影响。郭建春 [3]等基于有限单元法, 利用数值模 拟软件ABAQUS, 研究了三维尺度的缝间干扰现象。

目前针对该问题的研究大部分聚焦于常规储层或页 岩储层等弹性模量较高储层。煤层储层与这些储层相比岩 石塑性较大, 抗拉强度较小等特点 [4], 针对煤层这些特点, 本文运用数值模拟手段结合扩展有限元方法研究在煤层 中裂缝间相互干扰作用的影响, 并且定量的研究了射孔笶 数、簇间距以及簇间分布不均匀性对水力裂缝扩展的影响, 同时为现场应用实践提供相应的借鉴指导作用。

\section{2. 扩展有限元（XFEM）水力压裂法}

扩展有限元法 (XFEM) [5]在1999年被首次提出并用 于解决模拟过程中网格不连续性问题, 与传统数值模拟中 所用的有限元方法不同, 该方法通过在裂缝端部引入四节 点增强单元, 有效提高计算收玫性, 效解了决应力应变场
出现的奇异性问题。在扩展有限元中, 在解析解中引入裂 缝尖端渐进函数来准确计算裂缝端部应力场分布。

\section{1. 控制方程}

控制方程包括多孔介质流动平衡方程和连续性方程, 并通过固体力学本构关系对这些方程进行了补充。研究中 假设压裂多孔介质中流体流动遵循达西定律。

假设模型处于低速流动状态, 忽略液相相对加速度, 则多孔介质多相的线性动量平衡方程可以写为:

$$
\nabla \cdot \sigma+\rho b-\rho \ddot{\mathrm{u}}=0
$$

式中, $\ddot{\mathrm{u}}$ 为固相岩石加速度矢量; $b$ 为体积力矢量; $\rho$ 为多相系统平均密度; $\nabla$ 为矢量梯度算子。

液相通过变形等温多孔介质流动的连续性方程为:

$$
\frac{1}{Q_{w w}} \dot{p}_{w}+\frac{1}{Q_{w c}} \dot{p}_{c}+\alpha \nabla \cdot \dot{\mathrm{u}}+\nabla \cdot \dot{\mathrm{w}}_{w}+\nabla \cdot \dot{\mathrm{w}}_{g}=0
$$

式中, $\dot{\mathrm{u}}$ 为固相速度矢量; $\dot{\mathrm{w}}_{w}$ 和 $\dot{\mathrm{w}}_{g}$ 为液相和气相 达西速度矢量。

\section{2. 控制方程离散}

首先将控制方程采用扩展有限元法在空间上离散化, 然后采用广义Newmark格式在时间上离散化, 最后将平衡 方程和流动连续性方程的弱形式转化为离散形式。

在扩展有限元理论中，位移场渐进方程为:

$$
\mathrm{u}(x, t)=\sum_{I \in N} N_{u I}(x) \mathrm{u}_{I}(t)+\frac{1}{2} \sum_{I \in N^{e n r}} N_{u I}(x)\left(H_{\Gamma_{d}}(x)-H_{\Gamma_{d}}\left(x_{I}\right)\right) \tilde{\mathrm{u}}_{I}(t)
$$

式中, $N_{u l}$ 为有限元标准形状函数; $N$ 为离散区域所有 节点集合; $N^{e n r}$ 为增强节点集合; $N_{I}(t)$ 为标准位移自由 度; $\widetilde{u}_{I}(t)$ 为增强节点位移自由度。
为了对平衡方程和连续性方程进行离散化, 采用 Bubnov-Galerkin方法在同一近似空间中分别插入有限维 子空间测试函数。弱不连续多相方程的离散形式为:

$$
\begin{aligned}
& M_{u u} \ddot{U}+M_{u \ddot{u}} \ddot{\tilde{U}}+\int_{\Omega} B^{T} \sigma^{\prime \prime} d \Omega-Q_{u w} P_{w}-Q_{u c} P_{c}=F_{u}^{e x t} \\
& M_{u \ddot{u}}^{T} \ddot{U}+M_{\ddot{u} \tilde{u}} \ddot{\tilde{U}}+\int_{\Omega^{e v r}}\left(B^{e n r}\right)^{T} \sigma^{\prime \prime} d \Omega-Q_{u \tilde{w}} P_{w}-Q_{\ddot{u} c} P_{c}=F_{\tilde{u}}^{e x t} \\
& M_{w u} \ddot{U}+M_{w \tilde{u}} \ddot{\tilde{U}}+Q_{u w}^{T} \dot{U}+Q_{u w}^{T} \dot{\tilde{U}}+C_{w w} \dot{P}_{w}+C_{w c} \dot{P}_{c}+H_{w w} P_{w}+H_{w c} P_{c}=F_{w}^{e x t}
\end{aligned}
$$

式中, $M_{u u}, M_{u \widetilde{u}}$ 为系数矩阵。

\section{3. 裂缝扩展准则}

在裂缝扩展过程中, 模型每一次计算包含两个判定: (1)水力裂缝能否进行扩展; (2)如果水力裂缝能够扩展, 其向何处延伸。根据这两个判定, 需要制定两个相应的判
定准则, 一个用来判断裂缝能否向前扩展, 另一个判断裂 缝延伸路径, 即岩石破坏准则和损伤演化准则。

本文采用破坏准则为最大周向拉应力, 即当裂缝端部 最大周向应力达到材料的抗张强度时, 裂缝会在垂直于最 大拉应力的平面内径向延伸。 


$$
\left\{\begin{array}{c}
\sigma_{\theta} \\
\sigma_{r}
\end{array}\right\}=\frac{K_{I}}{4 \sqrt{2 \pi r}}\left\{\begin{array}{l}
3 \cos \theta+\cos \frac{3 \theta}{2} \\
\sin \frac{\theta}{2}+\sin \frac{3 \theta}{2}
\end{array}\right\}+\frac{K_{I I}}{4 \sqrt{2 \pi r}}\left\{\begin{array}{l}
-3 \sin \theta-3 \sin \frac{3 \theta}{2} \\
\cos \frac{\theta}{2}+3 \cos \frac{3 \theta}{2}
\end{array}\right\}
$$

式中, $\sigma_{\theta}$ 为周向应力; $\sigma_{r}$ 为剪应力。

模型损伤演化采用BK准则, 公式如下所示。

$$
G_{\text {equiv } C}=G_{I C}+\left(G_{I I C}-G_{I C}\right)\left(\frac{G_{I I}+G_{I I I}}{G_{I}+G_{I I}+G_{I I I}}\right)
$$

式中, $G_{\text {equiv }}$ 为临界断裂能释放率; $G_{I} 、 G_{I I} 、 G_{I I I}$ 分别 为法向、第一和第二切向裂缝能量释放率。

\section{3. 煤层多裂缝扩展计算模型建立及验证}

\section{1. 模型建立}

本文主要研究煤层水平井多簇射孔过程中, 裂缝间相 互干扰作用的影响因素, 故根据现场测井数据建立二维平 面计算模型如图1所示，本文所建立的二维正方形计算模 型边长为 $100 \mathrm{~m}$, 水力裂缝注入点位于射孔段中间, 射孔 长度为 $4 \mathrm{~m}$ 。整个模型采用孔压结构单元 (4节点位移-孔隙 压力单元）进行离散, 基于最小网格过渡原则将模型划分 为 40000 个四边形单元网格, 网格尺寸为 $0.5 \mathrm{~m}$ 。

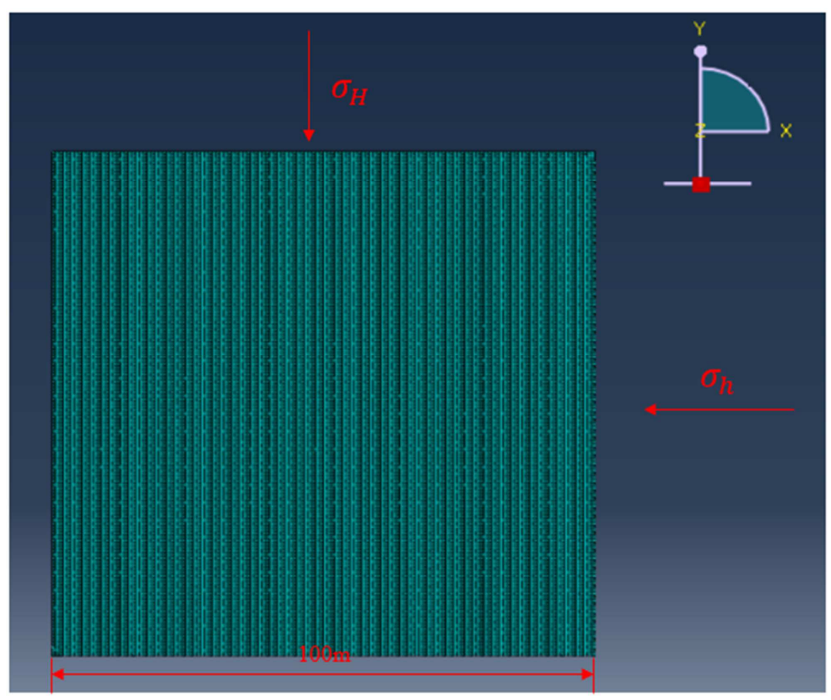

图1 煤层多琏计算模型示意图。

多缝扩展计算模型的部分参数如表 1 所示, 计算模型 参数主要包岩石的力学性质、储层物性以及施工参数等。 本文在此基础上, 分别改变模型射孔簇数、簇间距以及族 间分布不均匀性来研究这些参数对多缝扩展的影响 [6]。

表1 计算模型参数。

\begin{tabular}{lll}
\hline 输入参数 & 数值 & 单位 \\
\hline 杨氏模量 & 6 & $\mathrm{GPa}$ \\
泊松比 & 0.27 & 无量纲 \\
压裂液粘度 & 10 & $\mathrm{mPa} \cdot \mathrm{s}$ \\
\hline
\end{tabular}

\begin{tabular}{lll}
\hline 输入参数 & 数值 & 单位 \\
\hline 储层渗透率 & 0.43 & $\mathrm{mD}$ \\
初始裂缝长度 & 5 & $\mathrm{~m}$ \\
最大水平主应力 & 25.36 & $\mathrm{MPa}$ \\
最小水平主应力 & 20.50 & $\mathrm{MPa}$ \\
孔隙度 & 3.92 & 无量纲 \\
\hline
\end{tabular}

\section{2. 模型验证}

为验证模型的有效性, 研究过程中首先对Blanton实验 结果进行了模拟。模拟过程中应力场分布及岩石力学性质 严格遵循实验条件。通过对比注入压力曲线发现, 实验过 程中注入压力在 $75.3 \mathrm{~s}$ 时达到最大值 $14.12 \mathrm{MPa}$, 在数值模 拟过程中注入压力在 $77.6 \mathrm{~s}$ 时达到最大值 $13.86 \mathrm{MPa}$, 最大 注入压力误差为 $1.84 \%$ 。对误差原因进行分析后认为, 这 主要是由于实验过程中流体在管线中流动时存在沿程阻 力, 而在数值模拟过程中将该值忽略了。

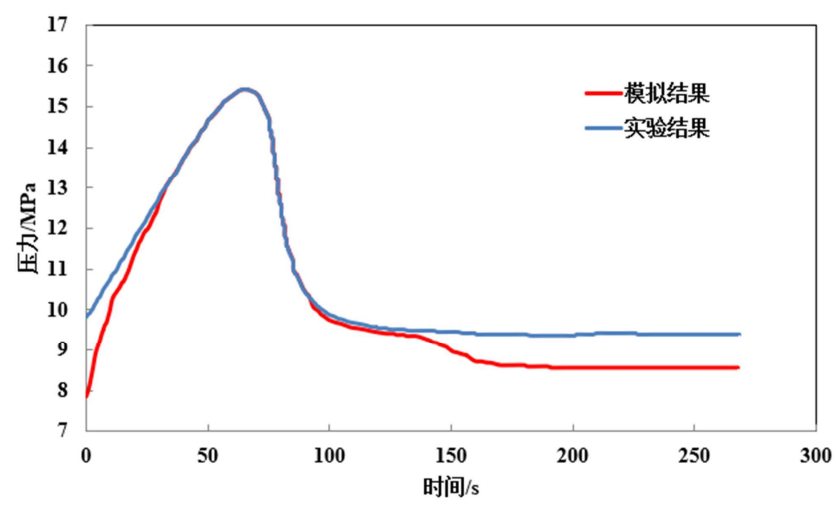

图 2 注入压力曲线模拟结果与实验结果对比。

\section{4. 模拟结果分析}

本文利用第二章所提理论以及第三章所建立的多裂 缝扩展计算模型, 结合ABAQUS数值模拟软件对射孔簇数、 簇间距以及簇间分布不均匀性进行研究。

\section{1. 射孔簇数}

本小结模拟分别建立单簇、双簇以及三簇射孔地层二 维多缝扩展计算模型, 如图3所示, 岩石内部预设纵向裂 缝, 模拟岩石的起裂和破坏。固定地层模型边界, 分别约 束边界法向位移, 同时为消除模型尺度对裂缝扩展的影响, 将模型边界设置为储层孔隙压力边界条件。定排量定压裂 液体系注入, 簇间距为 $10 \mathrm{~m}$, 模拟不同簇数对水力裂缝起 裂规律的影响。 


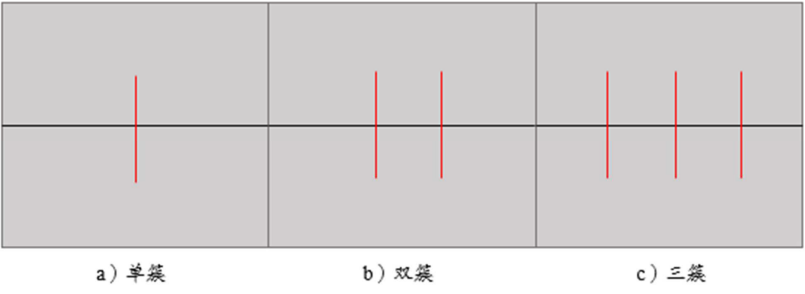

图3 不同簇数地层模型。

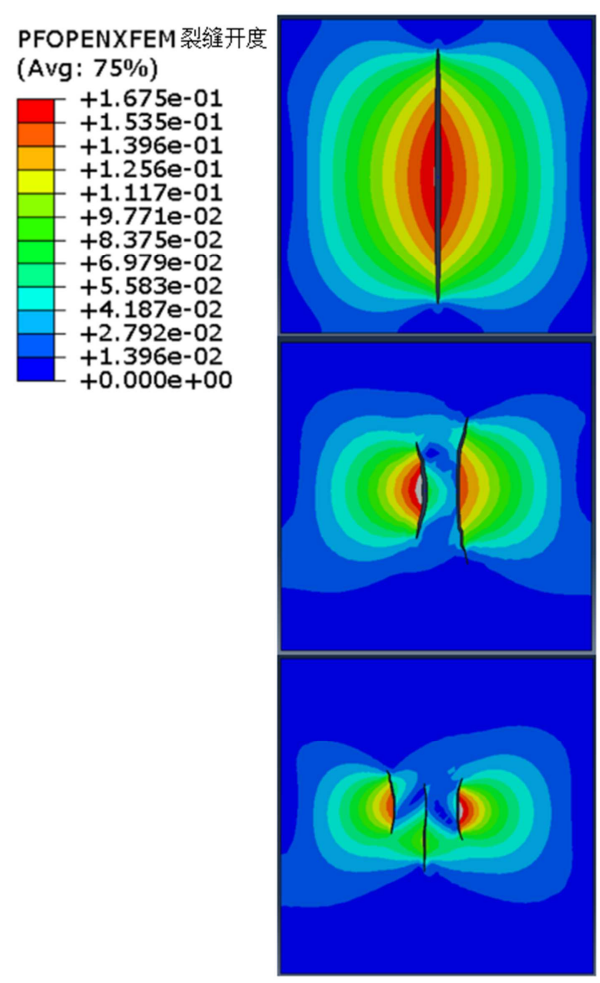

图4 不同簇数裂缝开度云图。

从模拟结果可以看出, 当采用单簇射孔时, 裂缝延伸 比较规则, 裂缝长度和宽度较大。当采用双簇方式射孔时, 裂缝宽度和长度均减小。当采用三簇方式射孔时, 中间裂 缝缝长缝宽较小, 两侧裂缝缝宽较大, 缝长较小。研究发 现地层中水力裂缝起裂后会改变局部地应力场, 影响周围 裂缝发育[7-11]。从双簇裂㖓扩展云图中可以看出, 两条 裂缝同时起裂时会相互影响, 在模型上部右侧裂缝抑制左 侧裂㖓延伸, 在模型下部左侧裂缝抑制右侧裂缝延伸。三 簇裂缝扩展云图可以更明显的表现出地应力干扰的影响, 研究表明, 当地层中存在多条平行裂缝同时扩展时, 两侧 裂缝会对中间裂缝产生压缩应力, 抑制中间裂缝缝宽, 这 就是应力阴影效应。从三簇裂缝扩展云图中可以看出, 中 间裂缝裂㖓宽度小于两侧裂缝宽度, 随着裂缝的扩展, 在 中间裂缝起裂点出甚至出现裂缝闭合现象, 这都是由于应 力阴影导致[12-16]。

\section{2. 射孔簇间距}

在三簇多缝扩展计算模型的基础上, 改变簇间距分别 为 $12 \mathrm{~m}$ 和 $24 \mathrm{~m}$ 时, 如图 5 , 模拟裂缝动态扩展规律, 研究裂 缝间相互干扰情况。

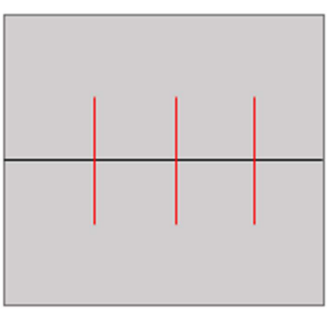

a) 䇲间距 $12 \mathrm{~m}$

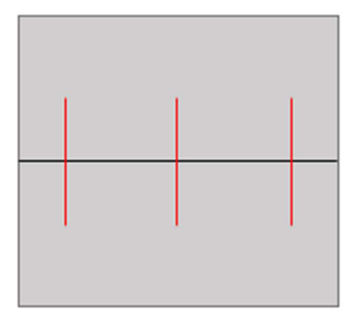

b) 䇲间距 $24 \mathrm{~m}$
图5 不同簇间距地层模型。

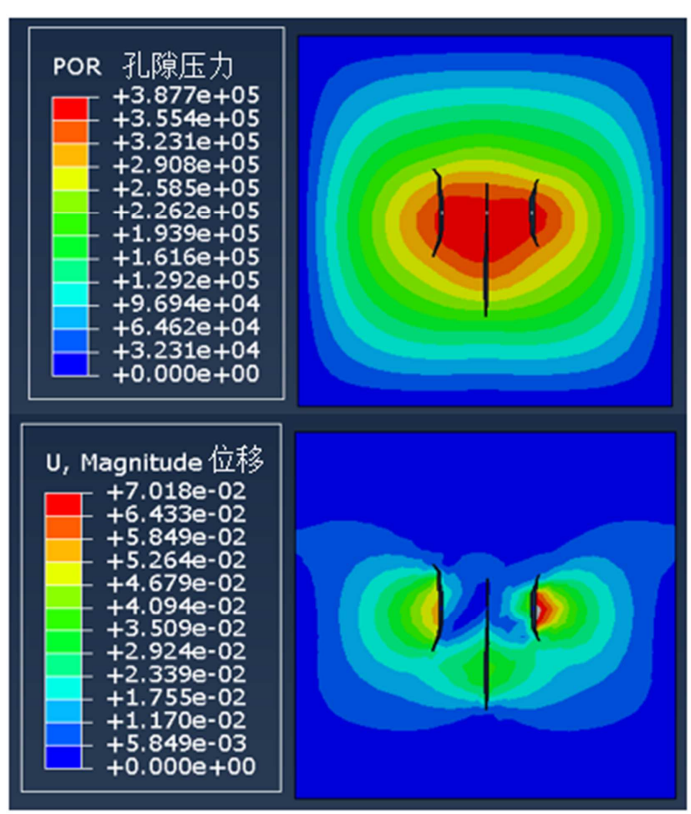

图6 簇间距 $12 \mathrm{~m}$ 裂缝扩展云图。

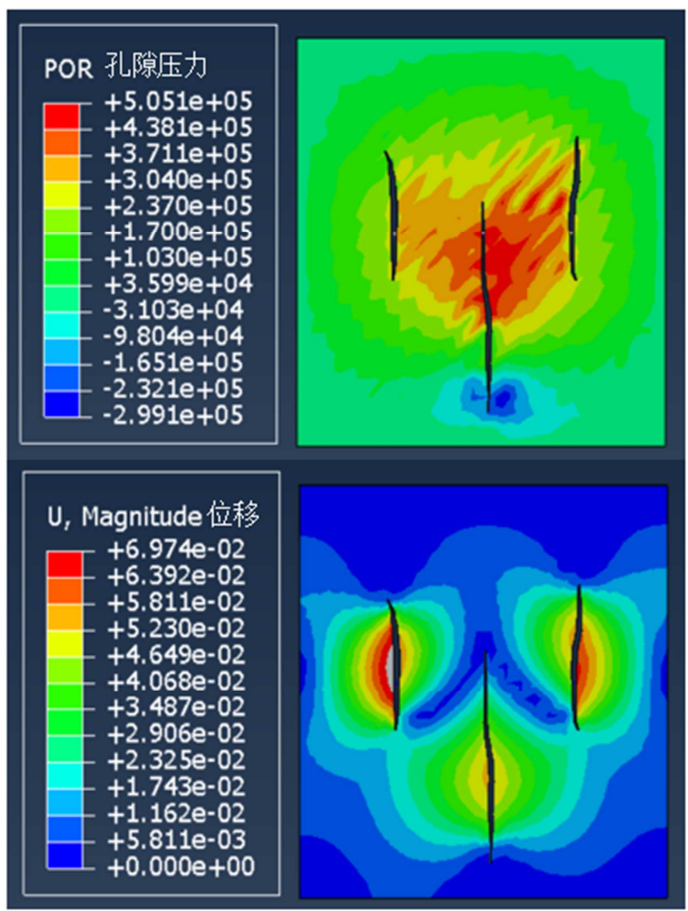

图7 簇间距 $24 \mathrm{~m}$ 裂缝扩展云图。 
由模拟结果可知, 当簇间距为 $12 \mathrm{~m}$ 时, 裂缝间相互影 响较为显著, 应力阴影较为明显, 两侧裂缝对中间裂缝延 伸的抑制效应较为明显。当族间距为 $24 \mathrm{~m}$ 时, 裂缝压制效 果明显减弱, 中间裂缝缝长缝宽均大于族间距为 $12 \mathrm{~m}$ 时的 裂缝缝长缝宽。

\section{3. 射孔簇间不均匀性}

在三簇多缝扩展计算模型的基础上, 改变中间簇位置, 分别模拟中间簇在 $1 / 2 、 1 / 3$ 、以及 $1 / 4$ 位置处, 如图 8 所示, 模拟裂缝动态扩展规律, 研究裂缝间相互干扰情况。

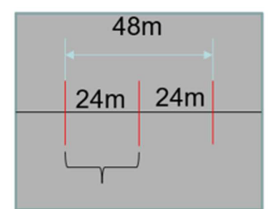

a) $1 / 2$ 位置处

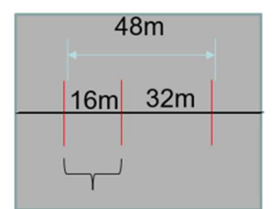

b) $1 / 3$ 位置处

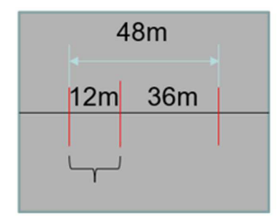

c) $1 / 4$ 位置处
图8 簇间不均匀性地层模型。

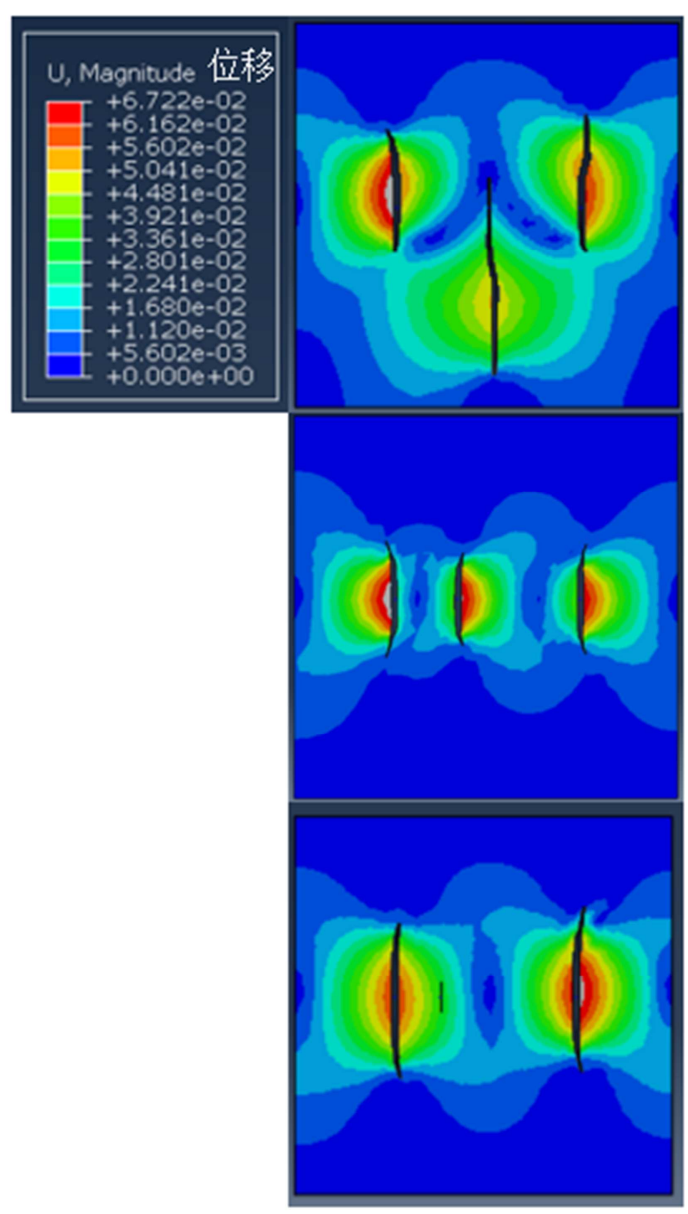

图9 簇间不均匀性裂缝扩展云图。

由模拟结果可知，中间簇位于 $1 / 2$ 位置时，中间裂缝 以及两侧裂缝发育较好, 并且中间裂缝缝长略大于两侧裂 缝。当中间族位于 $1 / 3$ 位置时, 中间裂缝发育受到抑制, 缝长缝宽小于位于 $1 / 2$ 位置处中间裂缝的缝长和缝宽。当 中间簇位于 $1 / 4$ 位置时, 中间裂缝发育完全受边缘裂缝抑
制, 无法形成有效裂缝, 边缘裂缝发育较为完善且左侧裂 缝缝宽大于右侧裂缝缝宽。

\section{5. 结论}

基于粘聚力本构模型和扩展有限元（XFEM）理论 [17-19], 建立了煤层多裂缝扩展模型, 利用该模型模拟了 不同射孔簇数、族间距以及簇间不均匀性对水力裂缝扩展 的的影响, 通过模拟发现:

1) 射孔簇数对裂缝起裂扩展影响较大, 当采用单簇 方式射孔时, 裂缝发育完善, 随着射孔簇数的增加, 由于 “应力阴影”的影响, 相邻裂缝发育逐渐被抑制, 当选取三 簇射孔时, 水力裂缝的长宽都远小于前两者。综合考虑缝 网展布范围以及主缝长度等因素后, 根据模拟结果可以看 出选用两簇射孔时压裂效果最佳。

2) 从模拟结果可以看出, 随着簇间距的增加, 裂缝 间“应力阴影”效果减弱, 缝长缝宽逐渐增加, 但是相应的 压裂时对地应力场的影响也逐渐增强。

3) 族间不均匀性严重影响了水力裂缝能否有效起裂, 当簇间均匀射孔时裂缝间相互抑制作用最弱, 当中间粗位 于 $1 / 4$ 位置时, 尽管簇间为 $12 \mathrm{~m}$, 但中间簇裂缝仍不能有效 起裂。

\section{参考文献}

[1] 王益山,王合林,刘大伟,袁孟雷,蒋海涛.中国煤层气钻井技 术现状及发展趋势[J]. 天然气工业,2014,34(08):87-91.

[2] Roussel NP, Sharna MM. Strategies to minimize fray spacing and stimulate natural fractures in horizontal completions [C]// SPE Annual Technical Conference and Exhibition. Society of Petroleum Engineers, 2011.

[3] Guo J, Lu Q, Zhu H, Wang Y, Ma L. Perforating cluster space optimization method of horizontal well mufti-stage fracturing in extremely thick unconventional gas reservoir [J].Journal of Natural Gas Seience and Engineering. 2015, 26:1648-1662.

[4] 李军,赵文光,李娜,洪国良.煤层形成背景与煤层气储层特征 [J].非常规油气,2015,2(02):27-33.

[5] 刘志强. 低渗透油气藏水力压裂数值模拟研究[D].中国科 学技术大学,2016.

[6] 魏波,陈军斌,谢青,张杰,王汉青,赵逸然.基于扩展有限元的 页岩水平井压裂裂缝扩展模拟[J].西安石油大学学报(自然 科学版),2016,31(02):70-75+81.

[7] 周圣吴. 煤层气多级压降排采工艺 [J]. 非常规油 气,2019,(06):63-67.

[8] 郭天鬼, 张士诚,刘卫来,赖文旭.页岩储层射孔水平井分段压 裂的起裂压力[J].天然气工业,2013,33(12):87-93.

[9] 张广明,刘合,张劲,吴恒安,王秀喜.水平井水力压裂的三维 有限元数值模拟研究 [J].工程力学,2011,28(02):101-106. 
[10] 张广明. 水平井水力压裂数值模拟研究[D].中国科学技术 大学,2010.

[11] 潘林华,张士诚,程礼军,陆朝晖,柳凯誉.水平井“多段分簇”压 裂簇间干扰的数值模拟 [J]. 天然气工业,2014,34(01):74-79.

[12] 艾军,肖传桃, 郭双,陈肯. 我国煤层气储层特征研究 [J]. 非常 规油气,2014,1(01):33-40.

[13] 张广清,陈勉. 水平井水力裂缝非平面扩展研究 [J]. 石油学 报,2005(03):95-97+101.

[14] 邓燕. 大位移井水力压裂裂缝起裂机理研究及应用[D].西 南石油学院,2003.
[15] 刘京. 基于扩展有限元的页岩水平井压裂裂缝扩展规律研 究[D].西安石油大学,2019.

[16] 冯依娜. 油气藏体积压裂缝网形成的力学研究及优化 [D]. 东北石油大学,2018.

[17] 李扬,邓金根,蔚宝华,刘伟,陈建国.储/隔层岩石及层间界面 性质对压裂缝高的影响 [J]. 石油钻探技 术,2014,42(06):80-86.

[18] 李杨. 全三维水力裂缝在砂/泥岩界面上扩展特性研究[D]. 东北石油大学, 2014.

[19] 熊文学, 袁旭, 惠涛, 王圣涛, 刘挺, 申超. 煤层气直井压裂参数 优化设计 [J].非常规油气,2016,3(04):95-103. 